\title{
Local Repair Mechanisms for On-Demand Routing in Mobile Ad hoc Networks
}

\author{
Michael Pan, Sheng-Yan Chuang and Sheng-De Wang \\ Department of Electrical Engineering \\ National Taiwan University \\ sdwang@ntu.edu.tw
}

\begin{abstract}
With the dynamic and mobile nature of ad hoc wireless networks, links may fail due to topological changes by mobile nodes. As the degree of mobility increases, the wireless network would suffer more link errors. Ad hoc routing protocols that use broadcast to discover routes may become inefficient due to frequent failures of intermediate connections in an end-to-end communication. When an intermediate link breaks, it is beneficial to discover a new route locally without resorting to an end-to-end route discovery. Based on the concept of localizing the route request query, we propose an efficient approach to repair error links quickly. The approach can apply to the Ad hoc On-demand Distance Vector (AODV) routing protocol. As an enhancement to AODV, the proposed approach leads to two routing protocols, called AODV-LRQ and AODV-LRT, which are aimed to efficiently repair the link errors. To evaluate the effects of the route repair, we define a factor, called bonus gain, as the ratio between the throughput increment to the routing overhead increment. Simulation results show that the proposed methods can get high bonus gain, that is, it can maintain the throughput as well as reduce the routing overheads.
\end{abstract}

keyword: local repair, route request broadcast, mobile ad hoc networks, $A O D V$, on-demand routing

\section{Introduction}

Wireless mobile ad hoc networks (MANET) are in general characterized by mobile nodes with limited energy, constrained computing capability and absence of any base stations. Mobile nodes communicate to each other by wireless communication with a limited transmission range and they are usually equipped with omnidirectional antenna. This results in multi-hop transmissions, which is quite different from cellular wireless networks. In this case, a source node may need intermediate nodes to forward packets to the destination node.

On-demand ad hoc routing protocols typically make use of request query broadcast to discover routes when a source-to-destination communication is needed. Among them, Ad hoc On-demand Distance Vector (AODV) and Dynamic Source Routing (DSR) [3] are the two most important routing methods. They are both on-demand reactive routing protocols. AODV is based on the distance-vector method [4], while DSR records all intermediate nodes in each source-destination pair. Due to the ever changing environment of MANET, the design of routing protocols is challenging and the resultant protocols are tending to be adaptive and dynamic. Mobility prediction is an approach to modeling the behavior of MANET and is aimed to enhance the reliable communication. In [5], the author uses a prediction table with pre-defined speed-role and some landmarks to predict mobility in the way-point graph [7]. However, in [6], the author predicts a node is stable or not by simply sending beacons to neighbor nodes. According to the address resolution protocol, we know that when a mobile node in dormancy would receive more beacons than it is in moving.

There are three types of important packets in AODV, that is, RREQ, RREP and RERR packets. The RREQ packet means a route request packet, which is sent when a node wants to construct a path to destination or when a link error occurs in the node. The RREP packet means a route reply, which is sent when it receives the RREQ packet and a route to destination has been found. The RERR packet means a route error packet, which is sent to the originator of the packet hop by hop when a node can't find a route to destination and every intermediate node that is in the previous successful path from the source to the current node will forward the RERR packet until the RERR packet is sent back to the source node of the packet.

AODV broadcasts a RREQ packet to the neighbors by the expanding ring search method, where an iterated increment of TTL (time-to-live) in the RREQ packet is used to discover the route to the destination. For example, a node will first send the RREQ packet with TTL=1 and then send 
RREQ packets with TTL $=2$ if it did not find a route to destination in the previous try. Finally the node will keep the TTL value until the end-to-end communication is finished.

Link errors usually happen in mobile ad hoc networks and they are also inevitable. One way to reduce the effects of link errors is to repair routes when link errors occur. Our route repair concepts are based on the localizing route request flooding and are aimed to reduce the control overheads as well as to keep the throughput in a transport layer protocol such as TCP. As can be seen from [14, 15, 16], although AODV is an adaptable routing protocol in the high mobility environment, it may incurs two much communication overhead when link errors happened. In this case, the RERR packets and RREQ packets will be triggered frequently by intermediate nodes involved in the route discovery. In the following, we will refer the communication overhead to the RREQ packets present in the network traffic.

The current implementation of [1] often incorporates a version of the local repair mechanism in AODV. However, the protocol has room to improve in the reduction of control overheads. In this paper, the goal is to reduce the control overheads as well as to keep the communication throughput under the TCP transport layer protocol. The remainder of this paper is organized as follows. Section 2 describes the related work. In Section 3 we will discuss the problems we concerned and propose the solution approach. In Section 4 we present our simulation study and experimental results. In Section 5 conclusions are included.

\section{Related Work}

A local repair procedure in AODV in initiated when nodes suffer link errors. The procedure broadcasts a RREQ (route request) packet to find a new route to the packet's destination as shown in Figure 2 instead of returning the error message to the source node of the packet as shown in Figure 1.

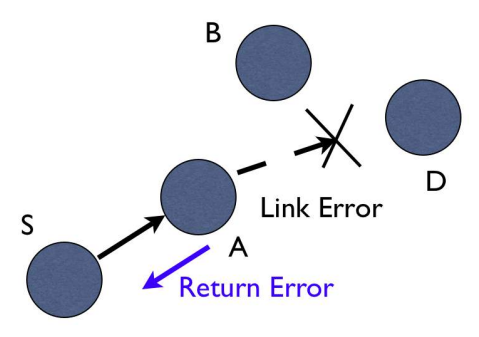

Figure 1. Link Error Occurs

Existing approaches [8] include a preemptive routing method that make use of proactive mechanisms to detect the link weakening so that a reliable route can be cho-

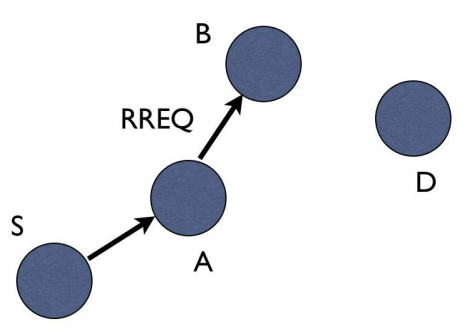

Figure 2. Repair RREQ

sen before the link breaks. In the preemptive approach, a node that detects a weakening link from one of it's neighbors will broadcast a Handoff packet with $\mathrm{TTL}=1$ to its neighbors. On receiving Handoff packet, each node will determine if any of its neighbors has links with more signal power than the current links. The information about link signal strength is kept by Neighbour Power List (NPL) and Power Difference Table (PDT). NPL records all neighbors' energy and PDT records the power difference between every two nodes in neighbors. As we can see, this approach costs a lot in storage for link power information.

Query localization techniques [2] are based on the concept of time locality. The time locality is kept track in a history path node list, Pold. Two kinds of repair mechanisms are discussed, that is, Exploiting Path Locality (EPL) and Exploiting Node Locality (ENL). In EPL, a node will broadcast a query with a counter and the accumulated path $(P)$ to at most $k$ nodes not in Pold. Figure 3 illustrates the above concept. In Figure 3(b), we can see that the number beside the node indicates the counter value. A node in ENL sends a query with Pold and a counter to neighbors when link error occurs. When a node receives a query, the nodes in ENL will drop the query it received if the counter in the query exceeds a threshold value $k$. The counter (initialized to zero) in the query packet will be incremented by a node that is not in Pold, otherwise the value of the counter will be kept. We set the ENL process with $k=2$ in the example of Figure 3.

In [9], a reliable route selection algorithm is proposed based on global positioning systems (GPS). Two zones are defined, a stable zone and a caution zone. A node will attempt to deliver RREQ packets to the other node in a nodepair when they are both in each other's stable zone. The key problem is how to define stable zone's radius. The approach in [9] is making use of GPS to get the position and speed of each node and thus define the radius of stable zone according the speeds of nodes.

A route discovery method called relative distance microdiscovery (RDMD) is proposed in [10]. The method is based on the estimation of relative distance between two 
ends and uses the value in TTL field in the RREQ packet. The relative distance is calculated by an iterated algorithm based on a specific route metric, velocity and last update time of nodes to localized the discovery range and assumes the velocity of the node is known. A similar and simple approach is also proposed in [11], where a two-hop broadcast is used to repair the link break.

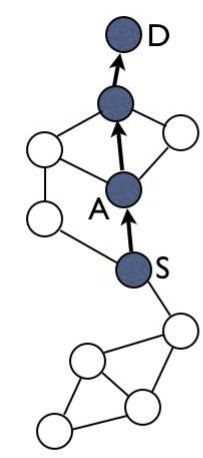

(a) Before route change

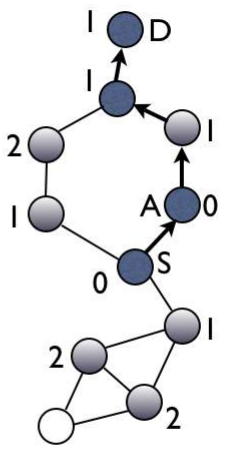

(b) After route change

\section{Figure 3. Illustration of the path locality prin- ciple with $k=2$}

In this paper, we will focus on improving the current implementation of local repair in AODV and keep the cost effective as well. The metric we will use to evaluate the local repair mechanisms is based on the number of successfully transmitted packets in a period. The cost we consider is the number of route request packets (RREQ) in the network traffic involved. To taking both the factors into account, we define a term called bonus gain to capture the concept.

We will apply the proposed protocol to AODV. Routing protocols in MANET has been widely investigated in literature such as [14], [15] and [16]. In [14], it shows that AODV has better performance than DSR in high mobility environments, and DSR has better performance than AODV-LL as described in [15]. AODV-LL is a variant of AODV with periodically broadcasting of Hello messages. AODV-LL is expected to have more overheads than AODV. This is also mentioned in [15]. As the result presented in [14], AODV will perform well in high mobility environments.

\section{Route Repair}

We conduct simple experiments to test the performance of the current implementation AODV-LR [13] of local repair method in NS-2 [12]. The experiments use default $\mathrm{TCP}$, transport layer protocol to send packets in a period of
300 seconds in NS-2 [12] simulator, and the network topology is consisted of 50 nodes randomly located in $1600 \mathrm{~m}$ $\mathrm{x} 500 \mathrm{~m}$ area. In order to simulate a high mobility environment, the maximum speed of each node is set to be $40 \mathrm{~m} / \mathrm{s}$ and the pause time of each node is 75 seconds. In AODV-LR, approximately $28.7 \%$ redundant RREQ (Route Request) packets were sent, but only $1.8 \%$ extra successfully transmitted packets are obtained.

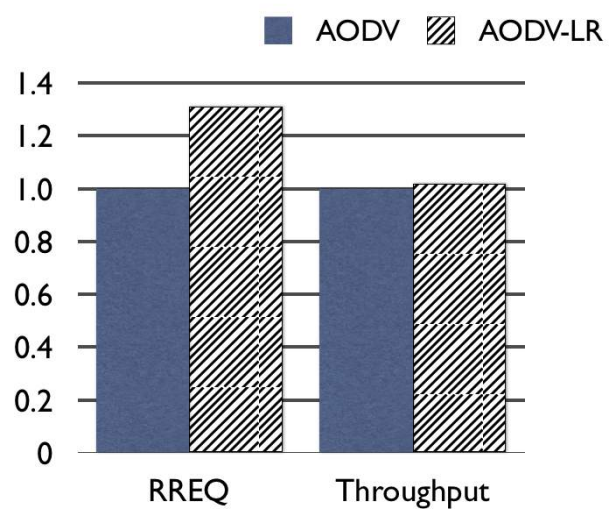

Figure 4. Cost vs. Performance

Figure 4 summarizes the results, where the left bar chart shows the normalized RREQ packets of AODV-LR with respect to AODV and the right bar chart shows the normalized throughput of AODV-LR to AODV. We can see that communication overheads increase so much as compared the throughput gained. Inspired by this observation, we are looking for an improved protocol to reduce the overheads and to keep the throughput as well.

In order to reduce the cost (RREQ packets) in AODVLR, we can decrease breadth or depth of the extent to which the repair mechanism applies in AODV-LR. Decreasing the depth of repair mechanisms means to limit the number of times a node is allowed to forward the repair route request. we can let each node carry out the route repair $k$ times in some period $T$. Decreasing the breadth of the repair mechanisms means we can limit TTL $=m$ in RREQ packets to localize the repair RREQs. The breadth and the depth constraints can apply in the same time to further localize the route request packets. In the following, we will propose a repair quota and an adaptive TTL approach based on the breadth and the depth constraints, respectively.

\subsection{Repair Quota}

In controlling the breadth, each node has a repair quota (RQ) which is initialized to $k$ in the start of a period. When a link error occurs, the node will determine if its repair quota is greater than 0 . If the node's RQ is greater than 0 , the node is allowed to carry out route repair operations and 
then decrease $k$ by one. In the other hand, the node will drop the RREQ packet received and send the RERR (route error) packet to the source node. A timer will periodically reset each node's RQ to $k$ as explained in Figure 5. We call this method AODV-LRQ.

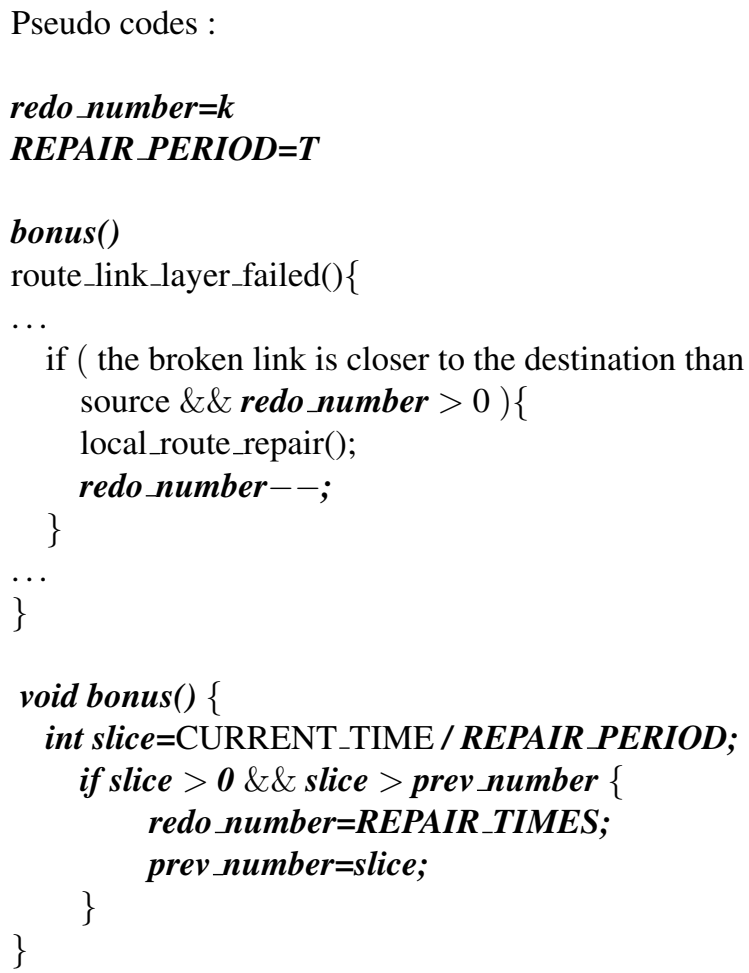

We denotes all variables with bold face above. Variable $\boldsymbol{r e d o}$ number represents the repair quota. Variable $\boldsymbol{R} \boldsymbol{E}$ PAIR_TIMES denotes the number of repair operations will be performed in each REPAIR PERIOD period. Function bonus() is a trigger timer that periodically resets the redo number. We divided the time by REPAIR_PERIOD and then we save time slice numbers in the variable, slice in function bonus(). The previous time slice number will be saved in the variable, prev_number and we will check if current time slice number, slice, is larger than the previous time slice number prev_number in the function bonus() before function local_route_repair is called. If the current time slice number is larger than the previous time slice number, the route repair operation is performed; otherwise the node will send a RERR packet to the source of the packet it just received like AODV without local repair does.

\subsection{Adaptive TTL}

To evaluate the effects of the breadth and the depth constraints, we choose to implement an adaptive TTL route repair algorithm, called AODV-LRT. As compared with the method of Exploiting Node Locality (ENL) proposed in [2], AODV-LRT adaptively adjusts the depth of the

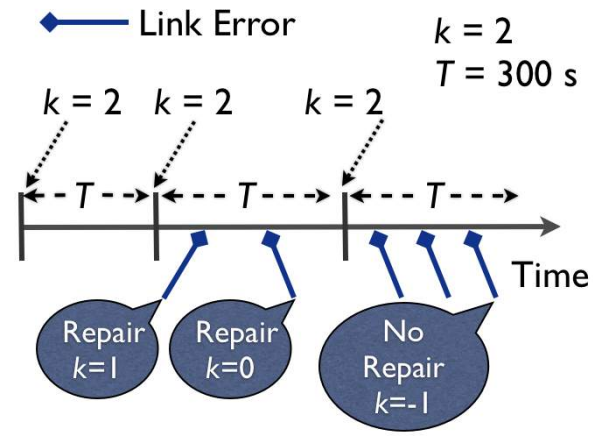

Figure 5. Repair mechanism with Timer and Repair Quota $k$

route request propagation without distinguishing between the nodes in an old path list and the other new nodes. We assume that the size of the network topology and the transmission rang of every nodes are known. We let TTL in repair time not exceed the half of the longest path. For example if the size of the netowrk topology is $1500 \mathrm{~m} \mathrm{x}$ $600 \mathrm{~m}$ and the transmission range in each node is $250 \mathrm{~m}$, the longest path will be 6 hops. In this kind of the scenario TTL in repair time will not exceed $6 / 2$ hops. The following pseudo codes set the TTL to an appropriate number.

Pseudo codes :

In sendRequest ()\{

‥

if this node is in repair

$\mathrm{TTL}=\min ($ longest path/2, previous $T T L)$ $\cdots$

\section{Simulations}

Network Simulator version 2 (NS-2) [12] is a wellknown simulator. We can do either wired or wireless network simulations in NS-2. We simulated basic AODV (AODV), AODV with local repair (AODV-LR), AODV with repair quota (AODV-LRQ) and AODV with limited TTL (AODV-LRT). We will discuss overall cost and performance of the four methods mentioned.

In our experiment we generated a wireless network consisted of 50 nodes randomly placed in $1600 \mathrm{~m} \times 500 \mathrm{~m}$ area and the simulation duration is 300 seconds. Each node's moving speed does not exceed $40 \mathrm{~m} / \mathrm{s}$ with 75 seconds pause time. Dependent on the network size, the parameters can be set as follows: $k=1$ and $T=300$ in AODC-LRQ; $m=3$ in 
AODV-LRT, since a packet with TTL $=3$ can go through half of the simulated network topology. During the simulation, total 20000 packets are transmitted using the TCP protocol.

\subsection{Overview Evaluation}

We evaluated the communication cost and the throughput of each method. The cost means the number of total RREQ packets received in a period, and total RREQ packets are the summation of RREQ packets in all nodes. The throughput means the number of total end-to-end successfully transmitted packets in the given period. To both consider the two factors, we define a bonus gain as the ratio between the throughput increment to the routing overhead increment. The performance of basic AODV will be used as a base in comparison. So the bonus gain will be computed as:

$$
\text { bonus gain }(R)=\frac{R_{t p}-A O D V_{t p}}{R_{R R E Q}-A O D V_{R R E Q}}
$$

where $R$ is a routing method and the subscripts $t p$ and $R R E Q$ stand for the throughtput and the number of RREQ packets, respectively. As we can see in Figure 6, apparently AODV-LRQ successfully reduced many RREQ packets when errors occur without degrading the throughput. In Figure 6 it seems that AODV-LRT performed almost as well as AODV-LRQ in the metric of communication overheads in terms of number of RREQ packets, but after taking a look at the bonus gain as shown Figure 7 we found AODVLRQ performed at least two folds better than AODV-LRT did. Furthermore, in the metric of bonus gain AODV-LRT performed two times better than AODV-LR. Overall, in terms of bonus gain of AODV-LRQ is five times better than AODV-LR. From this observation, decreasing the breadth to which the repair mechanism is applied is better than decreasing the depth.

\subsection{Detailed Evaluation}

In detailed evaluation of the performance, we want to take into consideration of the end-to-end distances in the communication. Average number hops is the average number of route length from source to destination that was generated by NS-2 in our experiment. We classified all end-toend transmissions by the successfully transmitted packet of AODV without local repairs and then compute the average of the hop number of each end-to-end transmission. The average number of hops could be used to model the relative distance of any pair of nodes. A small relative distance may represent they have a low relative speed or they are close enough to each other in the network.

As for the control overheads, in Figure 8 we can see both AODV-LRQ and AODV-LRT have less RREQ packets than
AODV-LR, and AODV-LRQ has less RREQ packets than AODV-LRT in most situations. From Figure 8 we know if we want to reduce more RREQ packets we should better limit repair quota in each node or set TTL less than 3 . As for the throughput, in Figure 9, we can see that more throughput can be obtained with local repairs in larger relative distances from 3.64 to 2.69 hops. In addition, if the relative distance is even more larger such as 4.27 hops, all protocols perform in the same way in this case since the no protocols can help in such fast changing environment.

It is interesting to compare the performances between AODV-LRQ and AODV-LRT, which are based on the constraints of breadth and depth, respectively. In terms of successfully transmitted packtes (i.e., the metric of throughput), AODV-LRQ performed well in large relative distances $(>=3.12$ hops) and AODV-LRT did better in small relative distances $(<=2.69$ hops), as can be seen from Figure 10. However, in terms of bonus gain, AODV-LRQ is better than AODV-LRT. This means that in general AODV-LRQ can have good cost effective results.

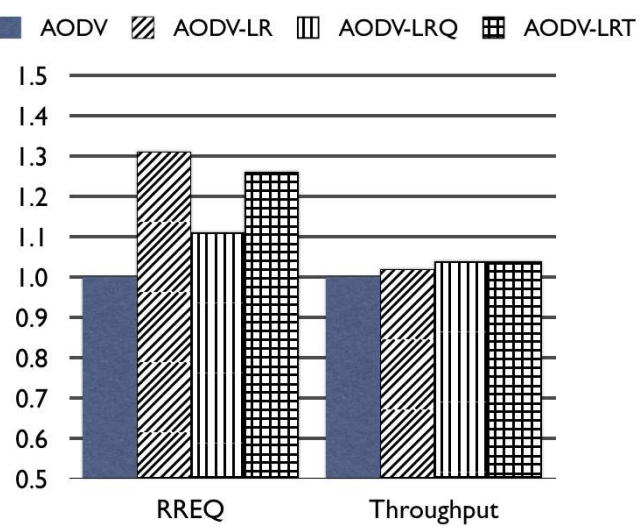

Figure 6. Overview cost and throughput

In the following, we did some comparisons over all variants of the proposed local repair mechanisms with different parameters. In Figure 11, LRQ-1-150 denotes the local repair approach with $\mathrm{k}=1$ and $\mathrm{T}=150$, which means the repair mechanism is launched once every 150 seconds in LRQ-1150 and AODV-LRQ uses local repair LRQ-1-300. In Figure 11 both AODV-LRQ and LRQ-1-150 get more throughput in terms of number of packets. Figure11 reflects the cost of original local repair is too high with respect to the methods we presented.

In order to see the performance of AODV-LRT in the large network topology environment we used different parameters from Table 1 and Table 2. Scenarios denoted in Table 1 and Table 2 represent a small and a lager topology, respectively. AODV-LRT obtained more successfully transmitted packets and better bonus gain in the large topology as can be seen in Figure 12. Thus, the strategy of adaptive 


\section{AODV-LR AODV-LRQ III AODV-LRT}

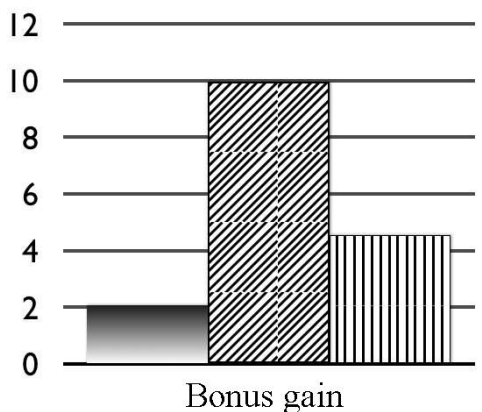

Figure 7. Increment of throughput over increment of cost

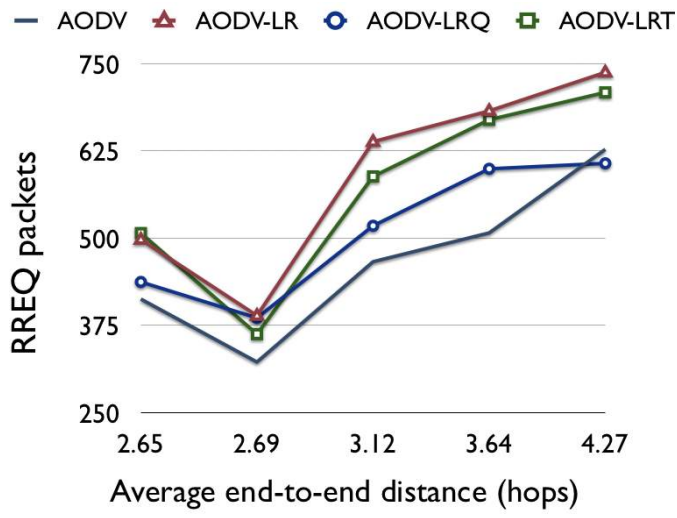

Figure 8. Illustration of overheads in RREQ packets vs. relative distances

TTL performs well both in the small topology and in the large topology.

Finally, we combined Repair Quota with Adaptive TTL at the same time to see the effects. A hybrid method, called AODV-LRQT, is created using AODV-LRQ with $k-T=1$ 300 and AODV-LRT. The results are shown in Figure 13 in Figure 14. In terms of successfully transmitted packets, AODV-LRQT performed only well when the route length is shorter than 3.12 hops. This is as expected as we can see that the hybrid method limits the overheads in two factors such that the throughput is hard to promote further. However, in terms of bonus gain, the hybrid method is competitive with the other methods as can be seen in Figure 14.

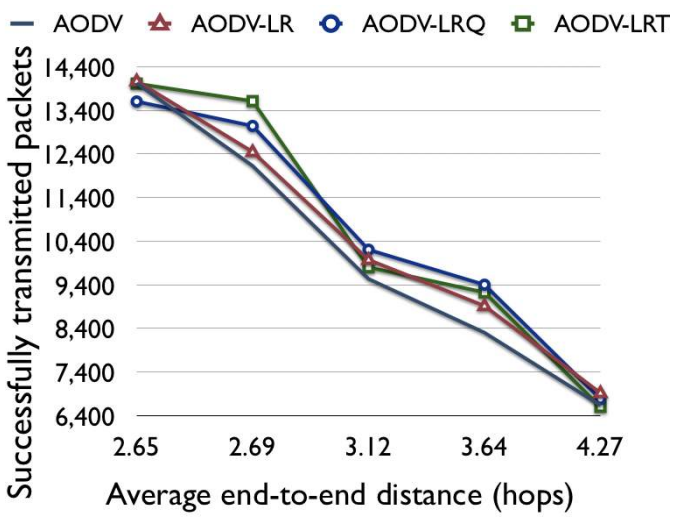

Figure 9. Illustration of the throughput vs. relative distances

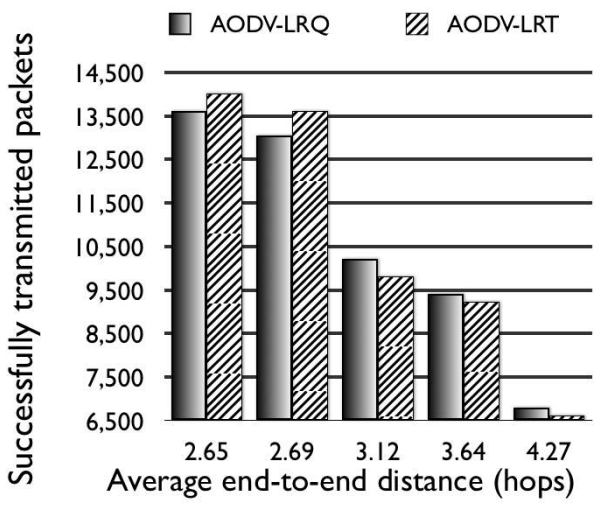

Figure 10. Detailed AODV-LRQ vs. AODV-LRT about transmitted packets

\section{Conclusion}

We have proposed a local repair approach to enhance the performance of on-demand ad hoc routing protocols. The concept of the proposed approach is based on the constraints of route repair requests in both the breadth and depth, which lead to the two routing protocols, called AODV-LRQ and AODV-LRT. To evaluate the effects of the route repair methods, we define a factor, called bonus gain, as the ratio between the throughput increment to the routing overhead increment. Simulation results show that the proposed methods can get high bonus gain as compared with the basic AODV-LR. In addition, we also found that the constraint in the breadth of route request floods can get better performance in most cases than the depth constraints. We also considered both the depth and breadth constraints for repair mechanisms at the same time. Hence, we get a new repair mechanism called AODV-LQRT (repair quota and adaptive 
Table 1. Simulation Scenario 1

\begin{tabular}{|c|c|}
\hline Parameter & Value \\
\hline Number of nodes & 50 \\
\hline Topology size & $1500 \mathrm{~m} \mathrm{X} 600 \mathrm{~m}$ \\
\hline Max. Speed & $40 \mathrm{~m} / \mathrm{s}$ \\
\hline Pause time & $75 \mathrm{~s}$ \\
\hline Simulation time & 300 \\
\hline
\end{tabular}

Table 2. Simulation Scenario 2

\begin{tabular}{|c|c|}
\hline Parameter & Value \\
\hline Number of nodes & 100 \\
\hline Topology size & $1800 \mathrm{~m}$ X $720 \mathrm{~m}$ \\
\hline Max. Speed & $40 \mathrm{~m} / \mathrm{s}$ \\
\hline Pause time & $75 \mathrm{~s}$ \\
\hline Simulation time & 300 \\
\hline
\end{tabular}

TTL) which combines AODV-LRQ and AODV-LRT.

In our experiment we set repair quota $k=1$ in a period of $T=300$ seconds in AODV-LRQ. In the future, we will do more experiments with different combinations of $k$ and $T$ in AODV-LRQ to find out the behaviors of AODV-LRQ more exactly. For example we could consider the new parameter called KT ratio, $k / T$ (repair quota over repair period) with various TTL to get a best KT with TTL in a given situation. Although it is hard to find a general repair mechanism that is suitable to every routing algorithms, existing routing algorithms can be improved based on our concept of decreasing depth and breadth of repair mechanism in MANET. If we want to design a repair mechanism to suit our needs, we can choose the method based on our concept of constraints of the breadth and the depth to which the route request broadcast is applied.

\section{Acknowledgments}

The work was partially supported by MediaTek Inc. Taiwan and partially supported by National Science Council, Taiwan, under the grant no. NSC-93-2213-E-002-123.

\section{References}

[1] C. E. Perkins and E. M. Royer, Ad-hoc On-demand Distance Vector Routing, In Proceedings of the 2nd IEEE Workshop on Mobile Computing Systems and Applications, pages 90-100, Feb 1999.

[2] R. Castaeda, S. R. Das and M. K. Marina, Query localization techniques for on-demand routing protocols in ad hoc networks, Wireless Networks, v.8 n.2/3, pages 137-151, March-May 2002.

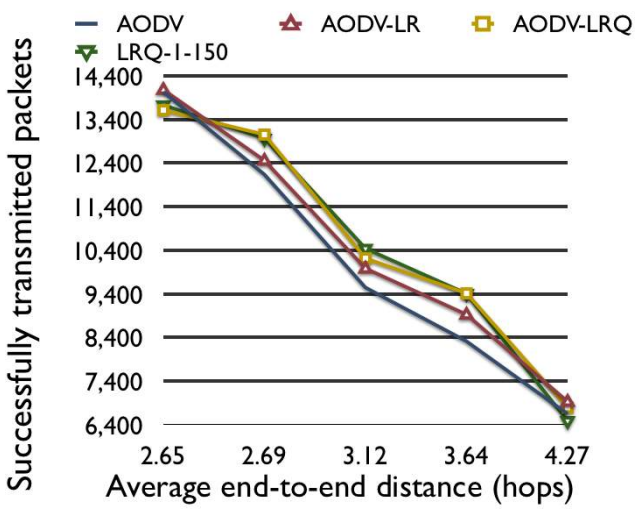

Figure 11. Effects of different Repair Quota parameters

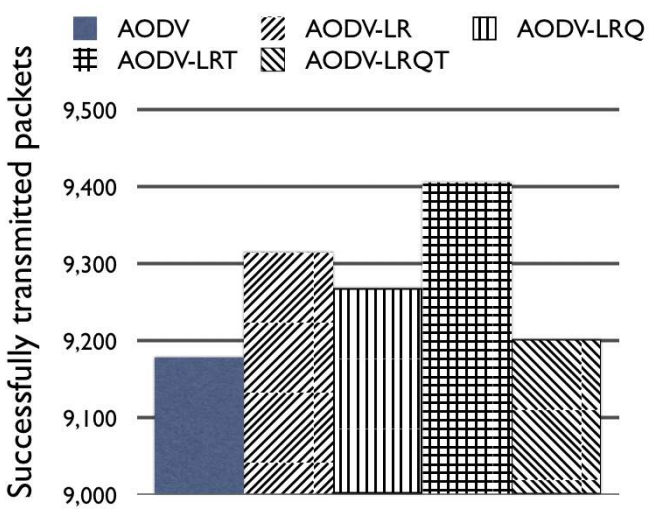

Figure 12. Overview of throughput in a large topology

[3] D. B. Johnson and D. A. Maltz, Dynamic Source Routing in Ad hoc Wireless Networks, Mobile Computing, 1994.

[4] C. Perkins and P. Bhagwat, Highly dynamic destination-sequenced distance-vector routing (DSDV) for mobile computers, in ACM SIGCOMM'94 Conference on Communications Architectures, Protocols and Applications, pages 234-244, 1994.

[5] Jian Tang, Guoliang Xue and Weiyi Zhang, Reliable Routing in Mobile Ad Hoc Networks Based on Mobility Prediction, Mobile Ad-hoc and Sensor Systems, pages 466-474, 2004

[6] Chai-Keong Toh, Associativity-Based Routing for Ad Hoc Mobile Networks, Wireless Personal Communi- 


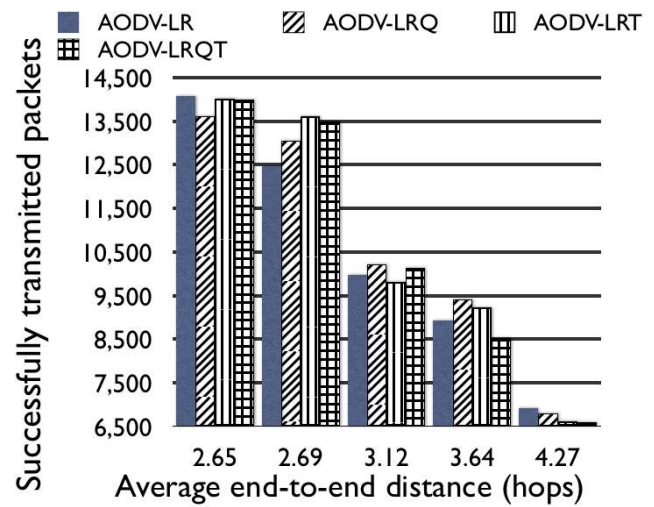

Figure 13. Detailed comparison over AODVLR AODV-LRQ AODV-LRT and AODV-LRQT
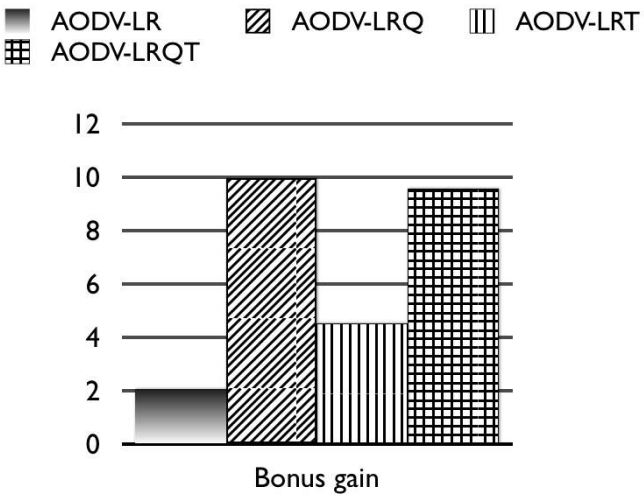

Figure 14. Bonus gain over AODV-LR AODVLRQ AODV-LRT and AODV-LRQT

cations: An International Journal, v.4 n.2, pages 103139, March 1997.

[7] J.-Y. Le Boudec and M. Vojnovic. The Random Trip Mobility Model, Last update: Nov 12, 2004. http://icalwww.epfl.ch/RandomTrip/

[8] P. Srinath, P. Abhilash, I. Sridhar, Router Handoff: A Preemptive Route Repair strategy for AODV, IEEE Intl. Conference on Personal Wireless Computing, New Delhi, December 2002.

[9] W.-I. Kim, D.-H. Kwon, and Y.-J. Suh, A Reliable Route Selection Algorithm Using Global Positioning Systems in Mobile Ad-hoc Networks, Proceedings of the IEEE International Conference on Communications (ICC'2001), June 2001.

[10] G. Aggelou and R. Tafazolli, RDMAR: A BandwidthEfficient Routing Protocol for Mobile Ad Hoc Net- works, Proceedings of the ACM International Workshop on Wireless Mobile Multimedia (WoWMoM), Seattle, WA, pp. 26-33, August 1999.

[11] 2003 G.P.Liu, PATCH: A novel Local Recovery Mechanism for Mobile Ad-hoc Networks,IEEE VTC, 2003

[12] The Network Simulator - ns-2, http://www.isi.edu/nsnam/ns/

[13] AdHoc@UU : : ImplementationPortal, Last updated: Feb 19, 2005. http://core.it.uu.se/AdHoc/ImplementationPortal

[14] C. Perkins, E.M. Royer, S.R. Das, and M.K. Marina, Performance Comparison of Two On-demand Routing Protocols for Ad Hoc Networks, IEEE Personal Communications, pages 16-28, Feb. 2001.

[15] J. Broch, D.A. Maltz, D.B. Johnson, A performance comparison of multi-hop wireless ad hoc network routing protocols, ACM MobiCom, pages 85-97, 1998.

[16] T. D. Dyer, R. V. Boppana, A comparison of TCP performance over three routing protocols for mobile ad hoc networks, Proceedings of the 2nd ACM international symposium on Mobile ad hoc networking \& computing, October 04-05, 2001, Long Beach, CA, USA 